\title{
HIGHLIGHTS
}

TARGETED THERAPIES

\section{Farletuzumab_-promising new agent in ovarian cancer}

Farletuzumab, a humanized monoclonal antibody that targets the folate receptor alpha (FRa), could potentially be used in the treatment of patients with relapsed ovarian cancer, according to the results of a recent open-label phase II trial.

Armstrong and colleagues investigated the efficacy of farletuzumab as a single agent or in combination with standard chemotherapy in patients with relapsed ovarian cancer following first-line therapy.

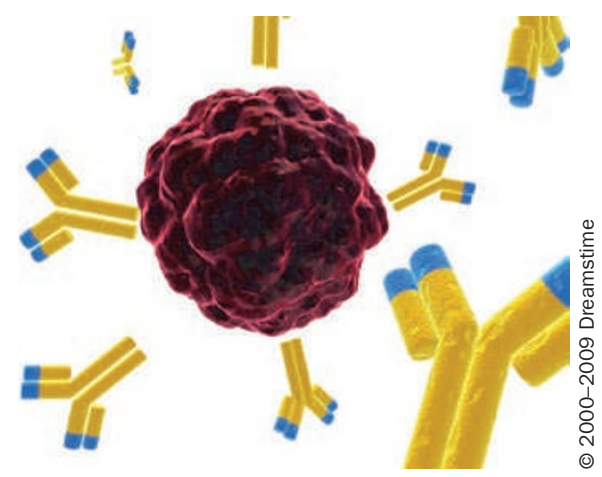

In total, 28 patients received farletuzumab and 46 patients received farletuzumab in combination with standard platinum and taxane chemotherapy.

The researchers reported preliminary data from 41 evaluable patients who received farletuzumab in combination with standard platinum and taxane chemotherapy. CA-125 levels were normalized in $90.2 \%$ of these patients. The overall response rate was $73.5 \%$ and the median progression-free survival was 10.3 months according to RECIST criteria. In addition, in more than $20 \%$ of patients the second progression-free interval was as long as or longer than the first.

Patients who received single-agent farletuzumab had disease stabilization or an improvement in outcome, but without an objective response or remission.

Farletuzumab did not increase toxic effects when administered with chemotherapy. The most common adverse events were pyrexia, headache and flushing.
Farletuzumab in combination with platinum and taxane increased the objective response rate of patients with relapsed ovarian cancer compared with chemotherapy alone. The researchers are excited about these promising preliminary data. Currently, there are two ongoing studies investigating the role of farletuzumab in combination with chemotherapy - a randomized global phase III study of standard platinum and taxane chemotherapy in patietns with platinum-sensitive ovarian tumors and a randomized phase II study of weekly platinum and taxane chemotherapy in patients with platinum-resistant disease.

\section{Vessela Vassileva}

Original article Armstrong, D. K. et al. Efficacy and safety of farletuzumab, a humanized monoclonal antibody to folate receptor alpha, in platinum-sensitive relapsed ovarian cancer subjects: preliminary data from a phase-2 study [abstract]. Eur. J. Cancer Suppl. 7, 450 (2009) 